\title{
A two-circular RNA signature as a noninvasive diagnostic biomarker for lung adenocarcinoma
}

\author{
Xiao-Xia Liu' ${ }^{1}$, Yi-E Yang ${ }^{2}$, Xiao Liu ${ }^{1}$, Meng-Yu Zhang ${ }^{1}$, Rui Li ${ }^{1}$, Yun-Hong Yin ${ }^{1}$ and Yi-Qing Qu ${ }^{1 *}$ (D)
}

\begin{abstract}
Background: Recently, circular RNAs (circRNAs) have been reported to be microRNA sponges and play essential roles in cancer development. This study aimed to evaluate whether circulating circRNAs could be used as diagnostic biomarkers for lung adenocarcinoma (LUAD).

Methods: The Gene Expression Omnibus (GEO) dataset was used to investigate differentially expressed circRNAs (DEcircRNAs) in paired LUAD tissues and adjacent nontumor tissues. The expression levels of the host genes were analyzed in The Cancer Genome Atlas (TCGA)-LUAD dataset, and the prognostic value was assessed using the KaplanMeier plotter. Quantitative real-time PCR (qRT-PCR) was performed to validate the expression of candidate circRNAs in the LUAD plasma and cells. The CCK8 assay was used to measure the function of circRNAs in cell proliferation. Competing endogenous RNA (ceRNA) network, gene ontology (GO) and Kyoto Encyclopedia of Genes and Genomes (KEGG) pathway analyses were performed to predict the possible mechanisms and functions of circRNAs in LUAD.

Results: Two upregulated and two downregulated circRNAs were identified as candidate circRNAs using bioinformatics analysis. qRT-PCR demonstrated that hsa_circ_0005962 was upregulated in LUAD plasma and cells, whereas hsa_circ_0086414 was downregulated. Receiver operating characteristic (ROC) curve analysis confirmed that a signature comprising the two circRNAs had good diagnostic potential, with an area under the ROC curve (AUC) of 0.81 $(P<0.0001)$. In addition, we observed that overexpression of plasma hsa_circ_0086414 was related to EGFR mutations $(P=0.001)$. Plasma hsa_circ_0005962 displayed significantly different expression before and after surgery in patients with LUAD $(P<0.0001)$. In vitro experiments suggested that hsa_circ_0005962 promoted LUAD cell proliferation. For future studies, we predicted the circRNA-miRNA-mRNA network for hsa_circ_0005962. Bioinformatics analysis revealed that hsa_circ_0005962 might be involved in LUAD development.
\end{abstract}

Conclusion: A circRNA signature was identified as a potential noninvasive biomarker for LUAD diagnosis.

Keywords: LUAD, CircRNA, Plasma, Diagnosis, Biomarker, Hsa_circ_0005962, Hsa_circ_0086414

\section{Background}

Lung cancer is the leading cause of cancer-related death worldwide [1], and $\sim 50 \%$ of these cancers are adenocarcinomas [2]. Despite improvements in diagnosis and treatment, the 5-year survival rate for lung cancer is approximately $18 \%$, mainly because most patients are

\footnotetext{
*Correspondence: quyiqing@sdu.edu.cn

${ }^{1}$ Department of Respiratory and Critical Care Medicine, Qilu Hospital of Shandong University, Jinan 250012, China

Full list of author information is available at the end of the article
}

diagnosed at an advanced stage [3]. Therefore, discovering accurate and sensitive biomarkers is imperative and will be beneficial for the early diagnosis of lung adenocarcinoma (LUAD).

Circular RNAs (circRNAs), which have a circular covalently closed structure, are derived from precursor mRNA back-splicing of thousands of eukaryotic genes, endowing circRNAs with higher tolerance to exonuclease digestion [4]. Over the past few years, numerous potential functions of circRNAs have been discovered, such as acting as miRNA sponges, modulating transcription 
and interacting with RNA-binding proteins (RBPs) [5]. In addition, previous research showed that circRNAs were involved in autophagy, apoptosis, the cell cycle and proliferation, suggesting that circRNAs might have great significance for human disease [6]. Moreover, increasing studies have suggested that circRNAs are closely associated with different cancers, including lung cancer [7, 8], gastric cancer [9], colorectal cancer [10], hepatocellular carcinoma [11, 12] and breast cancer [13].

Recently, circRNAs have emerged as novel biomarkers due to their characteristics of abundance, stability, conservation, and specificity $[6,14,15]$. Moreover, circRNAs can steadily subsist not only in cancer tissues but also in exosomes and the blood [16, 17]. A liquid biopsy is more convenient and less invasive than traditional biopsy for analysis of biomarkers in tumor tissues. Hence, circulating circRNAs may be suitable for use as potential biomarkers for cancer diagnosis. Tan et al. [18] and Hang et al. [19] confirmed that plasma circRNAs might be potential biomarkers for non-small cell lung cancer (NSCLC) patients. However, little is known about the expression of plasma circRNAs in LUAD patients.

In the present study, we aimed to identify and validate potential plasma circRNA biomarkers for the diagnosis of LUAD. We performed bioinformatics analysis to select candidate LUAD-related circRNAs and validated the expression of these circRNAs in LUAD plasma and cells using quantitative real-time PCR (qRT-PCR). CircRNAs have been proposed to act as competing endogenous RNAs (ceRNAs) [6]. CeRNAs can function as miRNA sponges through their binding sites to modulate miRNA activity on target genes [5]. To predict the possible mechanisms and function of circRNAs in LUAD, a ceRNA network was constructed and a functional analysis was performed.

\section{Methods}

\section{Selection of candidate circRNAs}

CircRNA expression profiles for LUAD were searched in the Gene Expression Omnibus (GEO) database, and GSE101586 was selected. Normalized microarray data were re-analyzed using the GEO2R tool for comparison between LUAD tissues and paired nontumor tissues. The CircBase [20] database was used to find host genes related to the circRNAs, and the CSCD [21] database was used to select LUAD-specific circRNAs. Furthermore, the expression levels of their host genes were analyzed in The Cancer Genome Atlas (TCGA)-LUAD dataset downloaded from the Cancer Browser (https:// xena.ucsc.edu/welcome-to-ucsc-xena/), and their prognostic value in LUAD was assessed using the KaplanMeier plotter [22].

\section{Patients and samples}

Peripheral blood was collected from 153 LUAD patients at the Qilu Hospital of Shandong University between April and July 2018 for plasma isolation. The patients we assayed were in different TNM stages of LUAD, of which 83 were in stage I, 13 in stage II, 30 in stage III, and 25 in stage IV. The diagnosis of each case was confirmed through histological examination. None of the patients had a prior history of other cancers or metastatic cancer from other sites or had received chemotherapy or radiotherapy prior to plasma collection. Paired preoperative and postoperative blood samples $(n=54)$ were collected from the same patients before surgery and on the seventh day after resection. The 54 healthy controls without a history of any cancer were individually matched to the LUAD cases by age and gender. This study was approved by the Ethics Committee of Qilu Hospital of Shandong University (KYLL-2013-097; 25 February 2014), and written informed consent was obtained from all patients or their guardians.

\section{RNA isolation and reverse transcription}

Total RNA was extracted from the patients' plasma using the TRIzol ${ }^{\mathrm{TM}}$ LS Reagent (Invitrogen, Carlsbad, CA, USA) according to the manufacturer's instructions. Whereas total RNA from the cells was isolated with the TRIzol Reagent (Invitrogen). The purity and concentration of the total RNA were evaluated with the NanoDrop Lite spectrophotometer (Thermo Scientific). The total RNA was subjected to cDNA synthesis using the PrimeScript ${ }^{\mathrm{TM}}$ RT Reagent Kit (Takara, Dalian, Liaoning, China). Briefly, $1000 \mathrm{ng}$ of total RNA was reverse transcribed into cDNA with random primers in a final volume of $20 \mu \mathrm{L}$.

\section{qRT-PCR}

The qRT-PCR was performed using the TB Green ${ }^{\mathrm{TM}}$ Premix Ex Taq ${ }^{\mathrm{TM}}$ II (TaKaRa) on the Applied Biosystems StepOnePlus Real-Time PCR System (Thermo Fisher Scientific). The PCR conditions were $95{ }^{\circ} \mathrm{C}$ for $30 \mathrm{~s}$, followed by 40 cycles at $95^{\circ} \mathrm{C}$ for $5 \mathrm{~s}$ and $60{ }^{\circ} \mathrm{C}$ for $30 \mathrm{~s}$ for each specific primer. Melting curves were generated at the end of amplification to ensure the specificity of the PCR products. Glyceraldehyde 3-phosphate dehydrogenase (GAPDH) was used as a reference gene, and the relative expression levels of the circRNAs were calculated using the $2^{-\triangle \Delta C T}$ method. The divergent primers for these circRNAs were obtained from BioSune Corporation (Shanghai, China).

\section{Cell culture and transfection}

All cell lines (A549, NCI-H1299, HCC827 and 16HBE) were purchased from Procell Life Science \& Technology Co., Ltd. (Wuhan, China) and confirmed by short tandem 
repeat (STR) profiling. HCC827 is a LUAD cell line with an acquired mutation in the EGFR tyrosine kinase domain (E746-A750 deletion), and 16HBE is a human bronchial epithelial cell line. The A549, NCI-H1299 and HCC827 cells were cultured in RPMI-1640 medium (Gibco, Invitrogen, Carlsbad, CA), and the 16HBE cells were cultured in DMEM (Gibco) supplemented with 10\% fetal bovine serum (FBS) and 1\% penicillin/streptomycin. All cell lines were grown in humidified air at $37{ }^{\circ} \mathrm{C}$ with 5\% $\mathrm{CO}_{2}$. The siRNA (si-hsa_circ_0005962, 5'-GAGACA ACUUGACAUCUCUTT-3') targeting the back-splice junction of hsa_circ_0005962 was synthesized by GenePharma (Shanghai, China). The A549 and H1299 cells were transfected with the siRNA using the Lipofectamine ${ }^{\circledR}$ 2000 Reagent (Invitrogen) according to the manufacturer's instructions. After transfection, the cells were processed to assess the knockdown activity by qRT-PCR or used for other experiments.

\section{Cell proliferation assay}

To measure whether hsa_circ_0005962 was involved in cell proliferation, we performed the CCK8 assay. A549 and H1299 cells were seeded into 96-well plates at a density of $5 \times 10^{3}$ cells per well after transfection and cultured for $24 \mathrm{~h}$. Cell proliferation was assessed using the Cell Counting Kit-8 (CCK-8; Beyotime, Shanghai, China). The CCK- 8 reagent was added to each well, and the cells were incubated at $37^{\circ} \mathrm{C}$ for $2 \mathrm{~h}$. The proliferation rates were determined at $0,24,48,72$, and $96 \mathrm{~h}$. The optical density was measured by a microplate reader set at $450 \mathrm{~nm}$. All experiments were repeated three times.

\section{CeRNA network analysis and function annotation}

Potential interactions between the circRNAs and miRNAs were predicted using CircInteractome [23] based on the TargetScan algorithm. In addition, miRNA-target interactions were predicted with TargetScan [24] and miRTarBase [25]. The circRNA-miRNA-mRNA network was constructed and visualized with the Cytoscape [26] software. To gain further insight into the functions, gene ontology (GO) and Kyoto Encyclopedia of Genes and Genomes (KEGG) pathway analyses were performed for the target genes using DAVID v6.8 [27], The significant enrichment results were accepted at a threshold $\geq 2$ gene counts with a $P$ value $<0.05$.

\section{Statistical analysis}

All statistical data were analyzed using SPSS 22.0 (SPSS, Chicago, IL, USA), GraphPad 7.0 (GraphPad Software, San Diego, CA, USA) and the R software 3.5.1. The differences between the tumor and normal groups were evaluated using the nonparametric Mann-Whitney U test. A paired $\mathrm{t}$ test or Wilcoxon matched-pairs signed rank test was applied to compare differences in circRNA expression between the preoperative and postoperative groups. A Chi square test was used to analyze the associations between circRNA expression and clinicopathological factors in the LUAD patients. Logistic regression analysis was performed to establish a LUAD diagnostic panel consisting of two circRNAs. Receiver operating characteristic (ROC) curve analysis and the area under the ROC curve (AUC) were used to assess the diagnostic value of the circRNAs. The cutoff value of the circRNAs was calculated using the Youden index (specificity + sensitivity -1 ). $P$ values $<0.05$ were considered statistically significant.

\section{Results}

\section{Identification of candidate circRNAs}

In the present study, we re-analyzed the microarray GSE101586 data [7] from the GEO database and detected differentially expressed circRNAs (DEcircRNAs) in paired LUAD tissues and adjacent nontumor tissues using the GEO2R tool. A total of 182 DEcircRNAs $(P<0.05$ and fold change $>1.5)$ were obtained. Among the 182 deregulated circRNAs, 28 were upregulated and 79 were downregulated (Fig. 1a). Next, we utilized the CircBase and CSCD databases to select LUAD-specific circRNAs. As a result, 23 LUAD-specific circRNAs were eventually detected. The differential expression of the 23 selected LUAD-specific circRNAs in the GSE101586 dataset is shown in Fig. 1b. Next, to identify candidate circRNAs, we analyzed the expression levels of their host genes in TCGA lung samples and the prognostic value of their host genes using the Kaplan-Meier plotter. Finally, two upregulated (hsa circ_0005962, hsa_circ_0003958) and two downregulated circRNAs (hsa_circ_0086414, hsa_circ_0001936) were identified as candidate circRNAs, because their host genes showed differential expression in the LUAD and adjacent normal tissues. We found that these circRNAs had prognostic value. The differential expression and survival analyses of the host genes of the four candidate circRNAs are shown in Fig. 1c, d, respectively.

\section{Validating the expression of the candidate circRNAs}

The expression of the four candidate circRNAs was verified in 153 primary LUAD and 54 normal plasma samples using qRT-PCR. The results showed that hsa_circ_0005962 was highly upregulated in LUAD $(P<0.0001$, Fig. $2 a)$ and hsa_circ_0086414 was downregulated $(P<0.0001$, Fig. $2 b)$, which was consistent with the microarray analysis results. However, hsa_circ_0003958 and hsa_circ_0001936 showed no significantly differential expression in the plasma between the LUAD patients and the normal controls $(P=0.63$, Fig. 2c; $P=0.50$, 

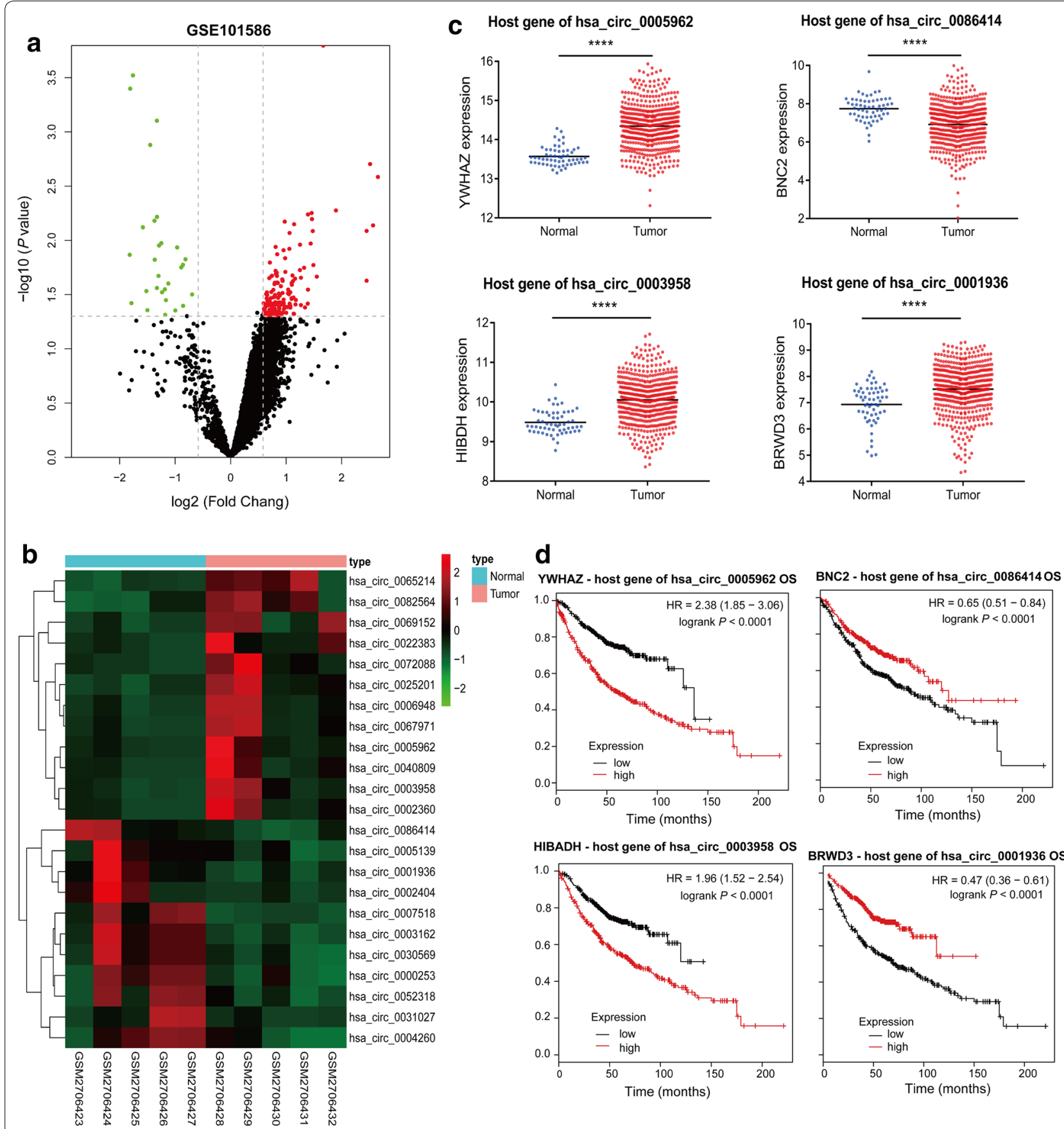

Fig. 1 Circular RNA (circRNA) expression profiles of lung adenocarcinoma (LUAD) and matched nontumor tissues. a Volcano plot showing the differential expression of circRNAs between the tumor and normal groups. $\mathbf{b}$ Heatmap of the differential expression of the 23 selected LUAD-specific circRNAs in the GSE101586 dataset. c The expression of host genes of hsa_circ_0005962, hsa_circ_0003958, hsa_circ_0086414 and hsa_circ_0001936 in The Cancer Genome Atlas (TCGA)-LUAD dataset. d Overall survival analysis for the host genes of the four circRNAs in LUAD patients was performed using the Kaplan-Meier plotter. Log-rank tests were used to determine statistical significance. ${ }^{* * *} P<0.0001$ 

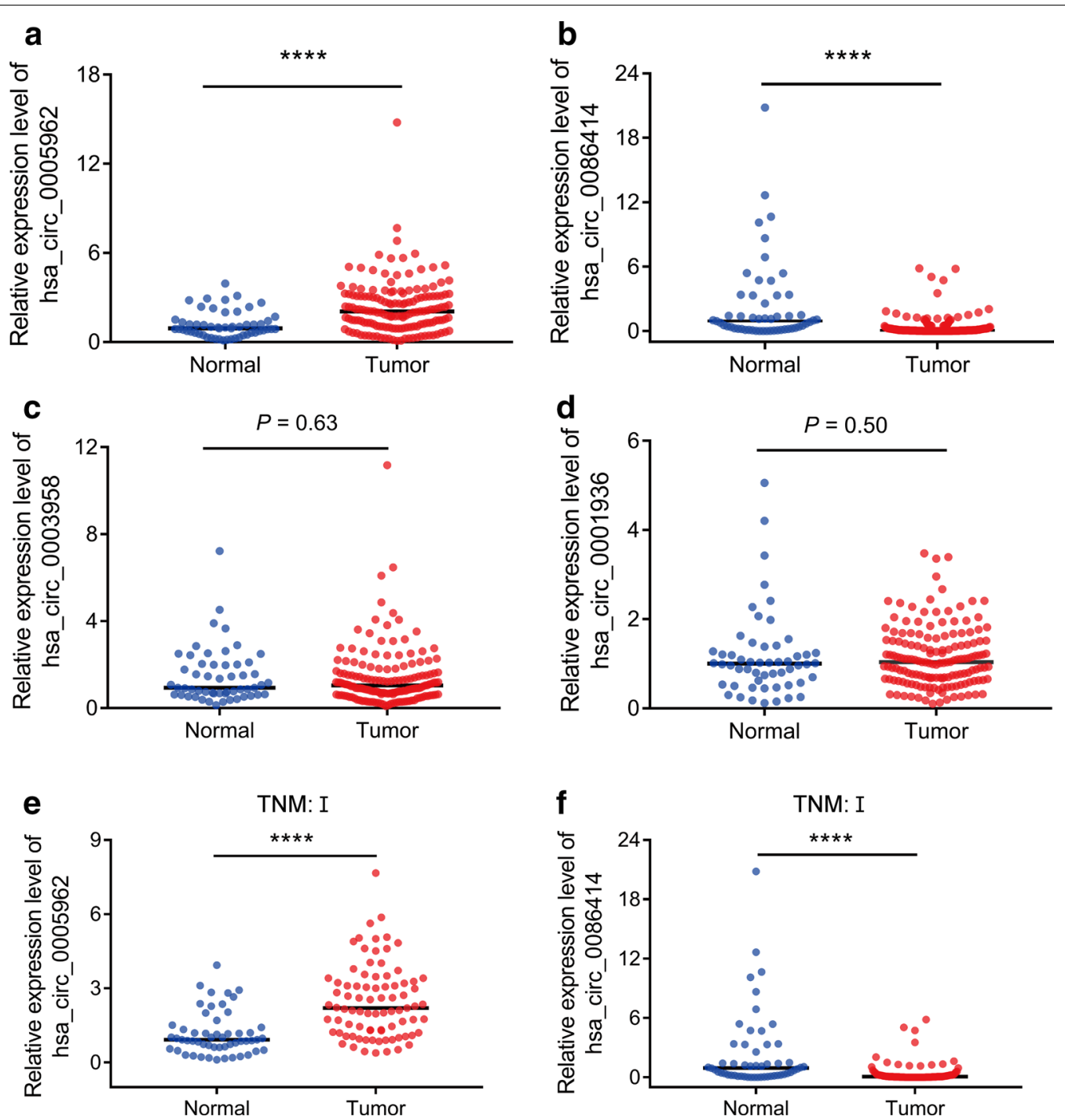

Fig. 2 Quantitative real-time PCR (qRT-PCR) analysis of the candidate circRNAs in the LUAD patients and normal controls. a Hsa_circ_0005962 expression was upregulated in the plasma of the LUAD patients compared with that of the normal controls. $\mathbf{b}$ Hsa_circ_0086414 was downregulated in the LUAD patients. Hsa_circ_0003958 (c) and hsa_circ_0001936 (d) were detected without significantly differential expression between the two groups. e Hsa_circ_0005962 was upregulated in LUAD patients with TNM stage I. f Hsa_circ_0086414 was downregulated in LUAD patients with TNM stage I. ${ }^{* * *} P<0.0001$

Fig. 2d). Furthermore, we found that hsa_circ_0005962 $(P<0.0001$, Fig. 2e) and hsa_circ_0086414 $(P<0.0001$, Fig. 2f) were differentially expressed in early LUAD patients and normal controls.

\section{Diagnostic value of hsa_circ_0005962 and hsa_ circ_0086414 for LUAD patients}

ROC curve analysis was used to investigate the diagnostic value of hsa_circ_0005962 and hsa_circ_0086414 in distinguishing LUAD patients from normal controls. As shown in Fig. 3a, the AUC of hsa_circ_0005962 was 0.73 and the optimal cut-off value was 1.21 , with a sensitivity of $71.90 \%$ and specificity of $72.22 \%(P<0.0001)$. For hsa_circ_0086414, the AUC was 0.78 and the cut-off value was 0.39 , with a sensitivity and specificity of $77.12 \%$ and $66.67 \%$, respectively $(P<0.0001)$. Notably, the combination of the two circRNA expression values provided the best discrimination, with an AUC of 0.81 , sensitivity of $77.80 \%$ and specificity of $72.22 \%$ $(P<0.0001)$. Furthermore, we analyzed the diagnostic performance of the two-circRNA signature in distinguishing LUAD patients with early TNM stage from healthy individuals. The AUC of the signature for LUAD patients with TNM stages I was $0.83(P<0.0001$, Fig. 3b). 

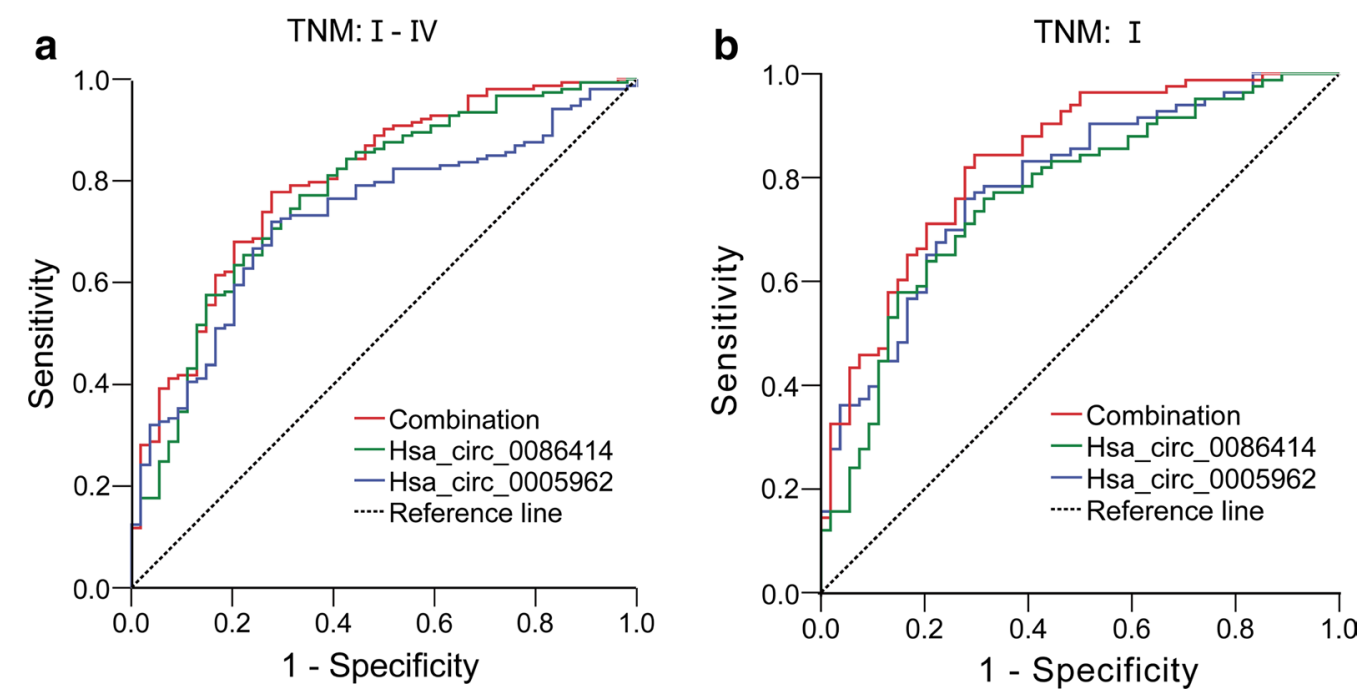

Fig. 3 ROC analysis of hsa_circ_0005962, hsa_circ_0086414 and the combination of the two markers for the diagnosis of LUAD. a TNM stages I-IV; Combination: The area under the ROC curve (AUC) 0.81, $P<0.0001$; Hsa_circ_0005962: AUC 0.73, $P<0.0001$; Hsa_circ_0086414: AUC 0.78, $P<0.0001$. b TNM stage I; Combination: AUC 0.83, $P<0.0001$; Hsa_Circ_0005962: AUC 0.79, $P<0.0001$; Hsa_circ_0086414: AUC 0.77, $P<0.0001$

\section{Correlation between expression of the two circRNAs and the clinicopathological characteristics}

An additional analysis was performed to assess correlations between the expression levels of the two circRNAs and the clinicopathological features of the LUAD patients. As indicated in Table 1, hsa_circ_0005962 and hsa_circ_0086414 expression was associated with gender $(P=0.024 ; P=0.046)$. Furthermore, a high plasma hsa circ_0086414 expression level was correlated with EGFR mutations $(P=0.001)$. However, we did not observe any association between circRNAs and the patients' ages, smoking history, tumor sizes, lymphatic metastasis, metastasis, CEA levels or TNM stages.

\section{Plasma hsa_circ_0005962 showed differential expression in LUAD patients before and after surgical resection}

Next, we examined hsa_circ_0005962 and hsa circ_0086414 expression in the plasma of LUAD patients before and after surgery. The results indicated that the hsa_circ_0005962 expression level was decreased in 42 of the $54(77.78 \%)$ LUAD patients after surgery $(P<0.0001$, Fig. 4a). However, no significant difference in hsa circ_0086414 expression was observed between the preoperative and postoperative stages $(P=0.11$, Fig. $4 \mathrm{~b})$.

\section{Hsa_circ_0005962 promotes the proliferation of LUAD cell lines in vitro}

To further explore the role of circRNAs in LUAD, we performed a preliminary in vitro experiment. First, we examined hsa_circ_0005962 and hsa_circ_0086414 expression in LUAD cell lines. The results showed that hsa_circ_0005962 was upregulated in the LUAD cell lines compared with that in human bronchial epithelial cells (Fig. 5a), whereas hsa_circ_0086414 was downregulated (Fig. 5b). Because hsa_circ_0005962 was significantly upregulated in LUAD plasma and cells, it was used as a target to investigate the role of circRNAs in LUAD tumorigenesis. A549 and H1299 cells were transfected with si-hsa_circ_0005962 or a negative control siRNA (si-NC). The qRT-PCR revealed that hsa_circ_0005962 expression was downregulated in LUAD cells by the siRNA compared with that of the cells treated with the si-NC (Fig. 5c). To explore whether hsa_circ_0005962 was involved in cell proliferation, we performed the CCK8 assay. We determined that knockdown of hsa circ_0005962 greatly suppressed A549 and H1299 cell proliferation (Fig. 5d). These in vitro experiments suggested that hsa_circ_0005962 promoted LUAD cell proliferation.

\section{Prediction of the ceRNA network for hsa_circ_0005962}

Based on the CircInteractome prediction, the top six miRNAs (hsa-miR-369-5p, hsa-miR-626, hsa-miR-326, hsa-miR-330-5p, hsa-miR-1265, and hsa-miR-622) targeted by hsa_circ_0005962 were identified. Then, we predicted the target mRNAs of these miRNAs in the TargetScan and miRTarBase databases. The 4 miRNAs and 203 target mRNAs are illustrated in Fig. 6. We predict that hsa-miR-1265 may directly target YWHAZ, which is the host gene of hsa_circ_0005962. Moreover, YWHAZ expression was increased in the LUAD 
Table 1 Association between the plasma circRNA expression levels and clinicopathological characteristics of LUAD patients

\begin{tabular}{|c|c|c|c|c|c|c|c|}
\hline \multirow[t]{2}{*}{ Characteristics } & \multirow[t]{2}{*}{$n=153$} & \multicolumn{3}{|c|}{ Hsa_circ_0005962 } & \multicolumn{3}{|c|}{ Hsa_circ_0086414 } \\
\hline & & Low $(n=43)$ & High $(n=110)$ & $P$ value & Low $(n=117)$ & High $(n=36)$ & $P$ value \\
\hline \multicolumn{8}{|l|}{ Age (years) } \\
\hline$<60$ & 74 & 22 & 52 & 0.665 & 58 & 16 & 0.59 \\
\hline$\geq 60$ & 79 & 21 & 58 & & 59 & 20 & \\
\hline \multicolumn{8}{|l|}{ Gender } \\
\hline Female & 93 & 20 & 73 & 0.024 & 66 & 27 & 0.046 \\
\hline Male & 60 & 23 & 37 & & 51 & 9 & \\
\hline \multicolumn{8}{|l|}{ Smoking } \\
\hline No & 99 & 23 & 76 & 0.097 & 72 & 27 & 0.089 \\
\hline Yes & 53 & 19 & 34 & & 45 & 8 & \\
\hline \multicolumn{8}{|l|}{ Tumor size } \\
\hline$\leq 3 \mathrm{~cm}$ & 99 & 25 & 74 & 0.907 & 75 & 24 & 0.956 \\
\hline$>3 \mathrm{~cm}$ & 42 & 11 & 31 & & 32 & 10 & \\
\hline \multicolumn{8}{|c|}{ Lymphatic metastasis } \\
\hline Negative & 111 & 27 & 84 & 0.091 & 87 & 24 & 0.366 \\
\hline Positive & 42 & 16 & 26 & & 30 & 12 & \\
\hline \multicolumn{8}{|l|}{ Metastasis } \\
\hline MO & 130 & 33 & 97 & 0.075 & 99 & 31 & 0.826 \\
\hline M1 & 23 & 10 & 13 & & 18 & 5 & \\
\hline \multicolumn{8}{|l|}{ TNM stage } \\
\hline । & 83 & 20 & 63 & 0.282 & 64 & 19 & 0.728 \\
\hline$\|$ and III and IV & 68 & 21 & 47 & & 51 & 17 & \\
\hline \multicolumn{8}{|l|}{ CEA } \\
\hline Negative & 75 & 16 & 59 & 0.146 & 56 & 19 & 0.337 \\
\hline Positive & 45 & 15 & 30 & & 37 & 8 & \\
\hline \multicolumn{8}{|l|}{ EGFR mutation } \\
\hline Negative & 26 & 10 & 16 & 0.287 & 25 & 1 & 0.001 \\
\hline Positive & 42 & 11 & 31 & & 25 & 17 & \\
\hline
\end{tabular}
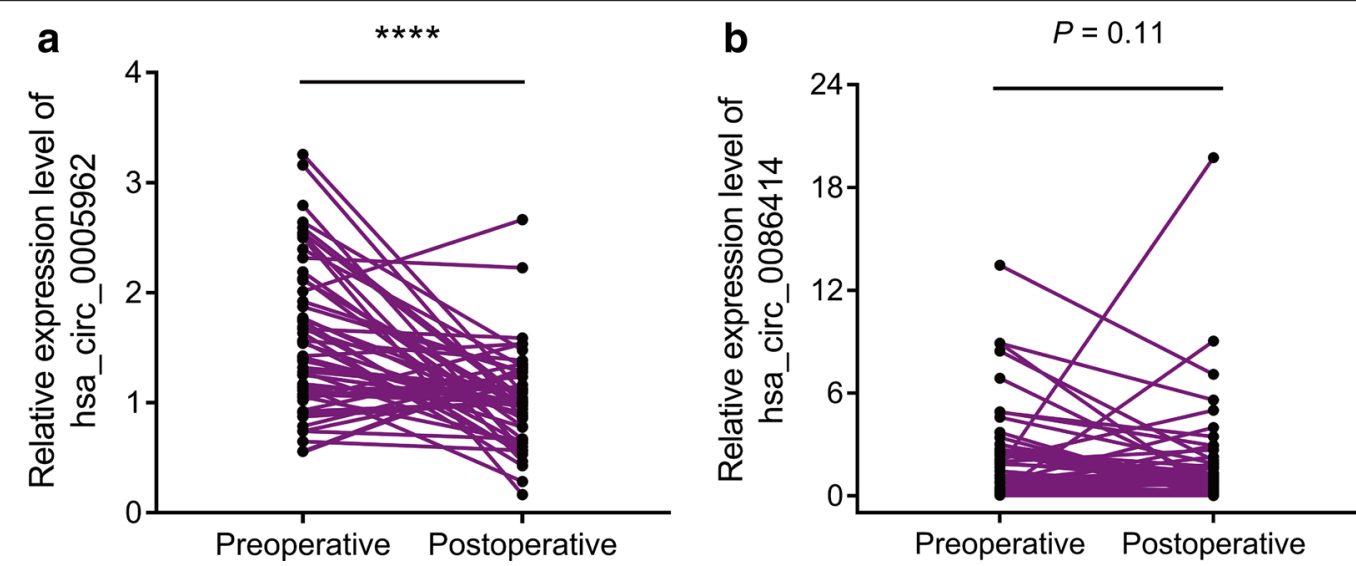

Fig. 4 Comparison of plasma hsa_circ_0005962 and hsa_circ_0086414 expression in preoperative and postoperative LUAD patients. a Upregulated hsa_circ_0005962 was detected at a lower level in the plasma samples after surgery. b No significant differential expression of hsa_circ_0086414 was detected in the patients before and after surgery. ${ }^{* * *} P<0.0001$ 

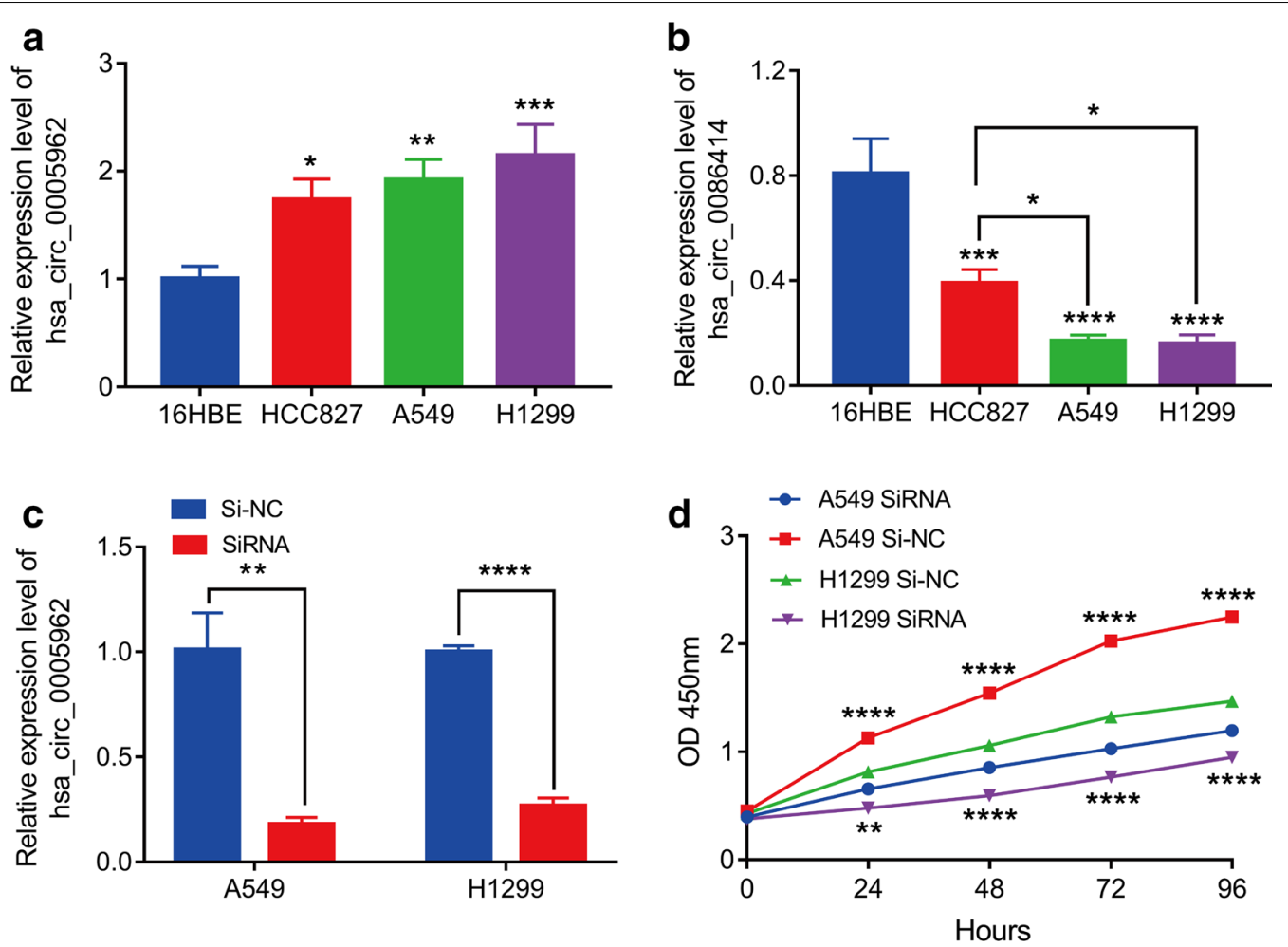

Fig. 5 Hsa_circ_0005962 promotes LUAD cell proliferation in vitro. a Hsa_circ_0005962 expression was upregulated in the LUAD cells (A549, H1299 and HCC827) compared to that of the normal human bronchial epithelial cells (16HBE). $\mathbf{b}$ Hsa_circ_0086414 was downregulated in the LUAD cells compared to that of the 16HBE cells and was differentially expressed between the LUAD cells with (HCC827) and without (A549 and H1299) EGFR mutations. c qRT-PCR analysis of hsa_circ_0005962 expression in A549 and H1299 cells after transfection with a specifically synthesized siRNA. d The CCK-8 assay showed that hsa_circ_0005962 promoted A549 and H1299 cell proliferation. ${ }^{*} P<0.05 ;{ }^{* *} P<0.01 ;{ }^{* * *} P<0.001 ;{ }^{* * * *} P<0.0001$

compared with the normal control tissues in TCGA dataset (Fig. 1c, $P<0.0001$ ). The microarray data from the Kaplan-Meier plotter indicated that patients with high YWHAZ expression levels had significantly poorer overall survival (Fig. 1d, $P<0.0001, \mathrm{HR}=2.38,95 \% \mathrm{CI}$ 1.85-3.06).

\section{Functional enrichment analysis of the hsa_circ_0005962 target genes}

Finally, GO analysis was conducted for hsa_circ_0005962. The top 10 significantly enriched biological processes (BPs), cellular components (CCs) and molecular functions (MFs) are shown in Fig. 7a. The main annotations included poly(A) RNA binding, protein binding, DNA binding and regulation of transcription and cell adhesion. Based on KEGG annotation, 18 pathways were identified, many of which were cancer-related, such as the p53 signaling pathway, pathways in cancer, PI3K-Akt signaling pathway, small cell lung cancer, chronic myeloid leukemia and cell cycle (Fig. 7b).

\section{Discussion}

Early diagnosis has great significance for the treatment and prognosis of LUAD. Unlike linear RNAs, circRNAs are expected to be novel candidates for biomarker detection, since they are more abundant and stable in body fluids (including serum exosomes, plasma and saliva) $[16,28,29]$. Recently, an increasing number of studies has noted that circRNAs can be used as biomarkers for cancer diagnosis [6]. Hence, in this study, we aimed to identify circulating circRNAs that could be used as biomarkers for the diagnosis of LUAD.

CircRNAs are derived from linear RNAs, most of which are produced by back splicing of exons [30]. A previous study demonstrated that most circRNAs were associated with their linear RNA expression during tumorigenesis [31]. In this study, we used a GEO dataset to investigate DEcircRNAs in LUAD and then selected the DEcircRNAs whose host genes were differentially expressed in TCGA-LUAD data and associated with the prognosis as candidate circRNAs. We speculated that these candidate DEcircRNAs might be involved in LUAD pathogenesis.

Furthermore, we validated the expression of the candidate circRNAs in the LUAD patient plasma. 


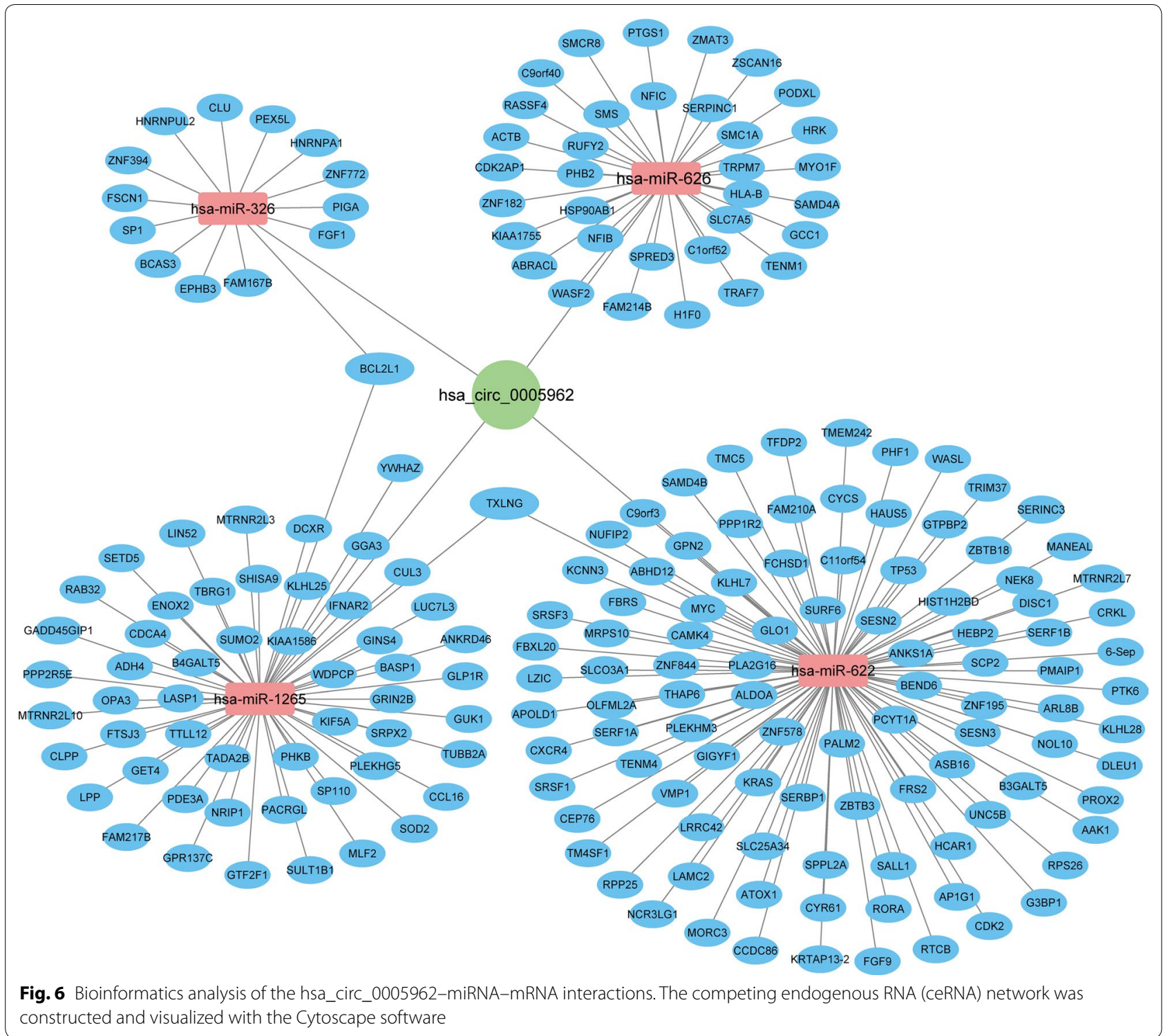

To the best of our knowledge, this report is the first on hsa_circ_0005962 and hsa_circ_0086414 expression in the plasma of patients with cancer. Our study utilized plasma samples due to their advantages of availability and noninvasiveness. Zhu et al. [32] found differential expression of plasma hsa_circ_0013958 between LUAD patients and healthy controls. However, their sample size was relatively small (30 LUAD cases and 30 healthy controls). In the present study, we verified the plasma circRNA expression levels in a relatively larger sample set (153 LUAD and 54 normal samples). We found that both hsa_circ_0005962 and hsa_circ_0086414 were differentially expressed in the plasma of LUAD patients and that the combination of these two molecules improved the diagnostic accuracy for LUAD. In addition, we found that hsa_circ_0005962 and hsa_circ_0086414 were differentially dysregulated in the early stage of LUAD, suggesting that they may be the promising diagnostic biomarkers for the early stage of LUAD. Although the other two candidate circRNAs showed no significantly differential expression in the plasma, we attributed this discrepancy to differences in the testing methods (microarray analysis vs qRT-PCR), sample types (tissue vs plasma) and sample sizes. The above results suggested that the two-circRNA signature could be used as a potential noninvasive biomarker for diagnosis of LUAD. 
a

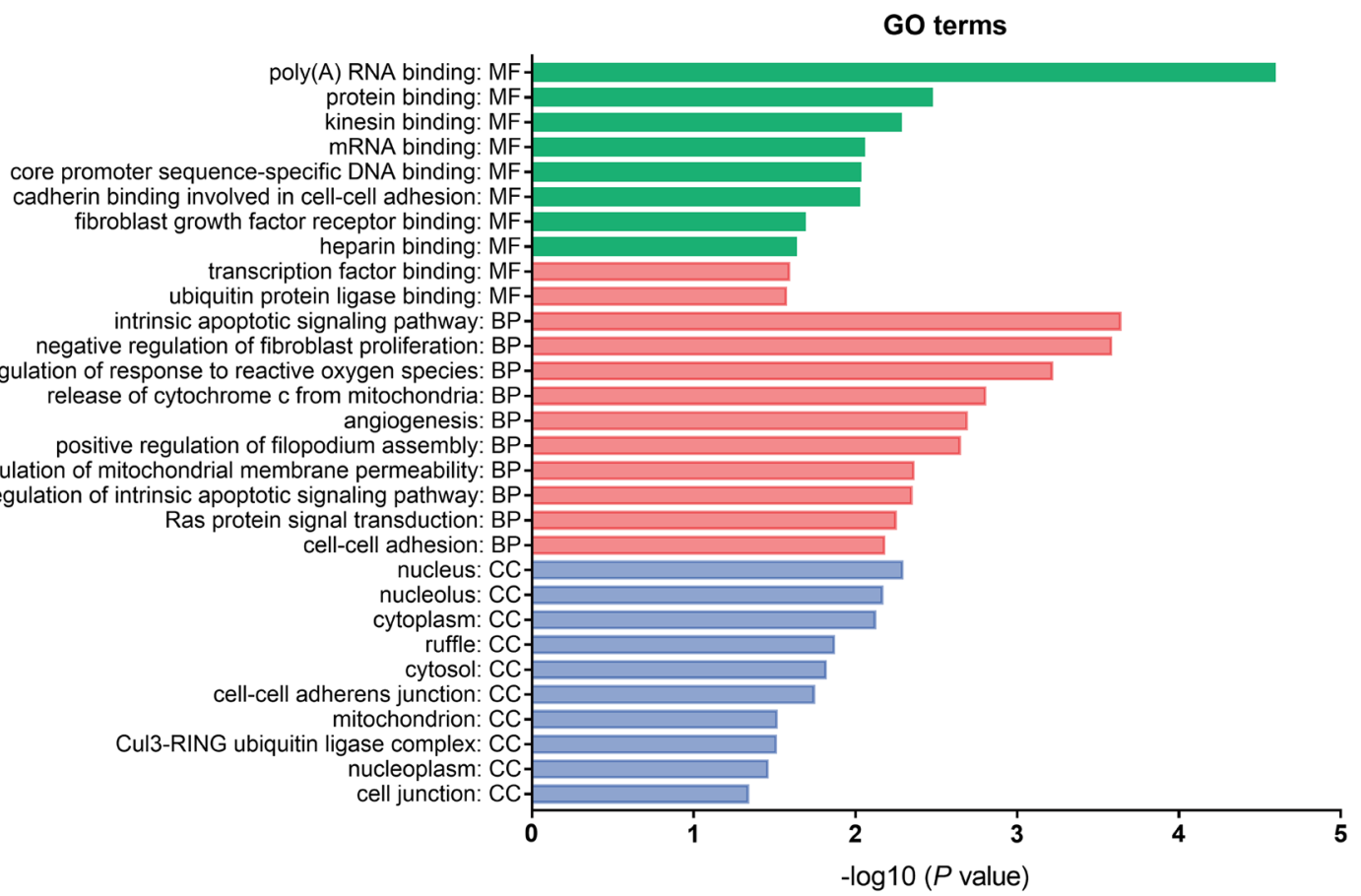

b

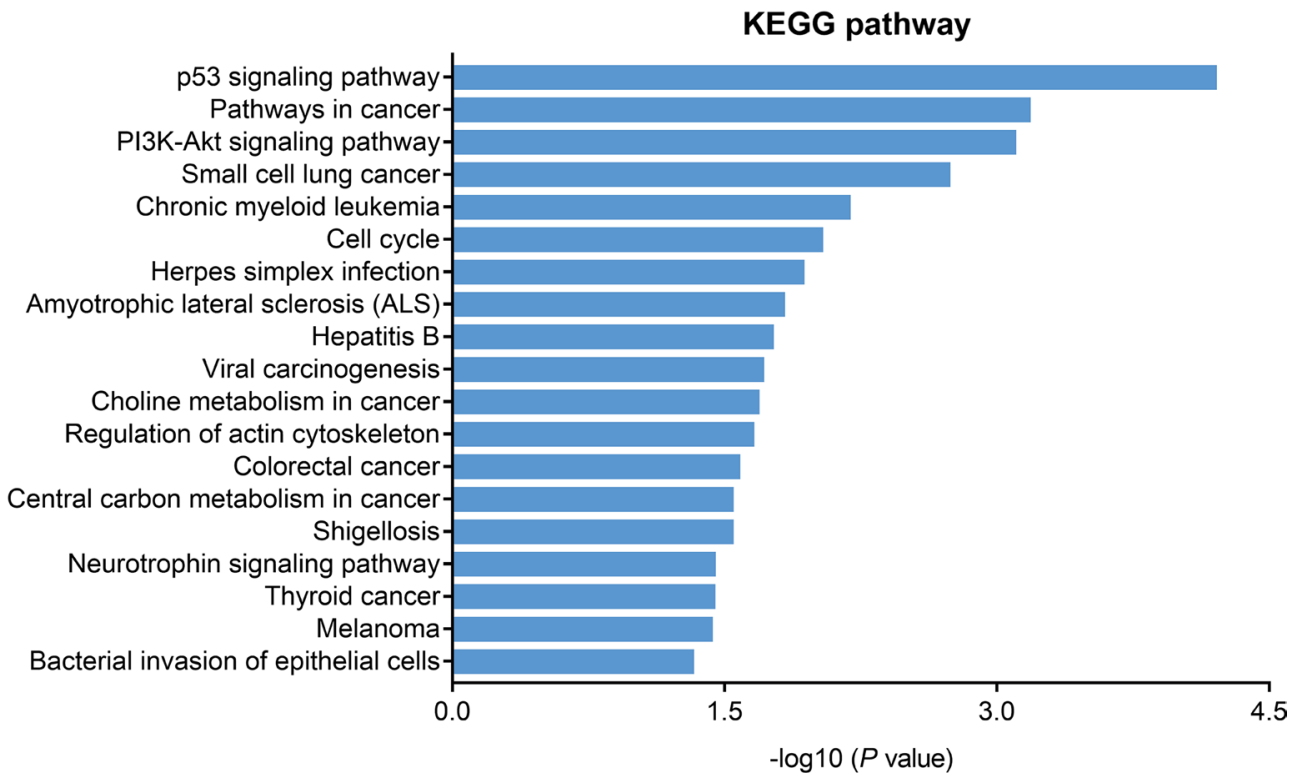

Fig. 7 Functional enrichment analysis for hsa_circ_0005962. a Gene ontology (GO) analysis of hsa_circ_0005962 based on the ceRNA network. The top 10 significantly enriched biological processes (BPs), cellular components (CCs) and molecular functions (MFs) are listed. b Kyoto Encyclopedia of Genes and Genomes (KEGG) pathway analysis of hsa_circ_0005962 based on the ceRNA network

Zhou et al. [31] detected a large number of circRNAs in plasma of cervical cancer patients showing differential expression before and after surgery, and some of these circRNAs were indicated as prognostic markers, suggesting that these plasma circRNAs may be associated with cancer progression. Li et al. [33] confirmed that the changes in plasma hsa_circ_0001017 and hsa_circ_0061276 expression before and after surgery were independent monitoring indicators for gastric cancer recurrence. In this study, we observed that hsa_circ_0005962 expression was decreased in postoperative LUAD patients compared to that in the preoperative patients, suggesting that 
it may be associated with the progression of LUAD. This decrease may be due to the decreasing release of tumorderived nucleic acids after tumor resection [34], resulting in significant changes in the plasma hsa_circ_0005962 levels before and after surgery. However, no significant difference in hsa_circ_0086414 expression was found between the preoperative and postoperative stages. It had been reported that the co-precipitation of circRNA with exosomes might be a possible mechanism for circRNA clearance [35]. Therefore, we may attribute this result to the increasing clearance of circRNA through exosomes, so that the expression of hsa_circ_0086414 was not significantly increased after surgery.

EGFR, which is the epidermal growth factor receptor, is a member of the ERBB receptor tyrosine kinase family that promotes cell survival, proliferation and invasion [36]. Mutations in EGFR are important drivers of NSCLC, and EGFR-targeted therapy can effectively improve the prognosis of patients with advanced NSCLC [37]. EGFR mutations occur mainly in adenocarcinoma, younger women, and never-smokers [38]. Surprisingly, we found that hsa_circ_0086414 was highly expressed in EFGR mutant patients compared to EGFR wild-type patients $(P<0.01$, Additional file 1 . Figure S1A), and was more highly expressed in female patients than male patients $(P<0.05$, Additional file 1 . Figure S1B). Previous studies showed that miRNAs could be involved in the development of EGFR mutations in LUAD [39-41]. Therefore, we hypothesized that hsa_circ_0086414 might contribute to EGFR mutation by binding miRNAs. To explore the possible mechanism, bioinformatics analysis identified 9 miRNAs might be the targets of hsa_circ_0086414, which were differentially expressed in patients with and without EGFR mutations (Additional file 2). Among the 9 miRNAs, hsa-miR-103a-3p was reported to inhibit the EGFR expression via EGFR/KRAS pathway [42]. However, the exact mechanism should be performed in the future study.

CircRNAs have been reported to regulate mRNA expression by competing for miRNAs [4]. For instance, circRNA ciRS-7 [43] and CDR1as [44] can bind to miR-7 and function as miRNA sponges. In this study, the CCK8 assay suggested that hsa_circ_0005962 might promote cell proliferation in LUAD. To further study this possibility, we predicted the hsa_circ_0005962-miRNA-target network and performed a functional enrichment analysis. As a result, 4 miRNAs (hsa-miR-626, hsa-miR-326, hsa-miR-1265, and hsa-miR-622) and their 203 target genes were identified. Previous studies showed that hsa-miR-326 inhibited SMO expression in glioma cancer and CD34(+) chronic myeloid leukemia cells and acted as a tumor suppressor miRNA by inhibiting the PI3 kinase pathway in glioblastomas [45-47]. In addition, hsa-miR-326 was reported to regulate lung cancer metastasis and invasion $[48,49]$. Research confirmed that hsa-miR-622 was downregulated in hepatocellular carcinoma, resulting in dysregulation of CXCR4 and KRAS $[50,51]$. Interestingly, YWHAZ, which is the host gene of hsa_circ_0005962, was predicted to be the target of hsamiR-1265. Moreover, bioinformatics analysis showed that YWHAZ was upregulated in LUAD and associated with the prognosis. Previous research certified that YWHAZ (also known as 14-3-3zeta) was overexpressed in NSCLC and promoted cancer progression $[52,53]$. These findings supported the hypothesis that hsa_circ_0005962 might function as a sponge for hsa-miR-1265, thus increasing expression levels of YWHAZ and promoting tumorigenesis of LUAD. Functional enrichment analysis revealed that its target genes were involved in several cancer-related pathways, including the p53 signaling pathway, pathways in cancer, PI3K-Akt signaling pathway, small cell lung cancer, chronic myeloid leukemia and cell cycle. This evidence indicated that hsa_circ_0005962 might act as a miRNA sponge to promote the development of LUAD and further research on its mechanism is worthwhile.

Finally, to better understand the clinical application of circRNAs, we searched the clinical trials using the term "circRNAs" from the website https://clinicaltrials.gov/, and https://www.who.int/ictrp/en/. Interestingly, there were recruiting clinical trial for circRNAs as biomarkers for prostate cancer (ChiCTR1800019529), acute myocardial infarction (ChiCTR1800019218) and acute lung injury (NCT03766204) in the year 2018. However, there were no ongoing clinical trials for circRNAs biomarker for lung cancer. Our present study provided the evidence for circulating circRNAs as noninvasive biomarkers for lung cancer.

\section{Conclusions}

In conclusion, the present study validated the significant upregulation of hsa_circ_0005962 and downregulation of hsa_circ_0086414 in LUAD plasma and cells, suggesting the potential for use of this two-circRNA signature as a novel noninvasive biomarker for LUAD diagnosis. Furthermore, we found that the hsa_circ_0086414 levels were associated with EGFR mutations. Hsa_circ_0005962 had different expression in the plasma of LUAD patients before and after surgery, and in vitro experiments and in silico analysis indicated that it might be involved in the development of LUAD. 


\section{Additional files}

Additional file 1: Figure S1. Correlation between hsa_circ_0086414 expression and EGFR mutations and gender. (A) Hsa_circ_0086414 was highly expressed in EGFR mutant patients compared to EGFR wild-type patients. (B) Hsa_circ_0086414 was more highly expressed in female patients than male patients. ${ }^{*} P<0.05$; ${ }^{* *} P<0.01$.

Additional file 2: Table S1. The predicted miRNA targets for hsa_ circ_0086414 in the CircBank database. Table S2. The miRNAs that were significantly differentially expressed between the EGFR mutated and EGFR wild-type LUAD patients in the GSE119268 dataset. Table S3. Nine miRNAs might be the targets for hsa-circ-0086414 and that were differentially expressed in patients with and without EGFR mutations.

\section{Abbreviations}

circRNAs: circular RNAs; LUAD: lung adenocarcinoma; GEO: Gene Expression Omnibus; DEcircRNAs: differentially expressed circRNAs; TCGA: The Cancer Genome Atlas; qRT-PCR: quantitative real-time PCR; ceRNA: competing endogenous RNA; GO: gene ontology; KEGG: Kyoto Encyclopedia of Genes and Genomes; ROC: receiver operating characteristic; AUC: area under the ROC curve; RBPs: RNA-binding proteins; NSCLC: non-small cell lung cancer; GAPDH: glyceraldehyde 3-phosphate dehydrogenase; BP: biological process; CC: cellular component; MF: molecular function.

\section{Authors' contributions}

$X-X L$ designed the research, performed the experiments, analyzed the data, and wrote the paper; $\mathrm{Y}-\mathrm{HY}$ and $\mathrm{RL}$ provided the patient samples; $\mathrm{XL}$ and $\mathrm{M}-\mathrm{YZ}$ performed the data analysis and interpreted the data; Y-QQ assisted with the study design and revised the manuscript. All authors read and approved the final manuscript.

\section{Author details}

${ }^{1}$ Department of Respiratory and Critical Care Medicine, Qilu Hospital of Shandong University, Jinan 250012, China. ${ }^{2}$ Department of Clinical Laboratory, Qianfoshan Hospital of Shandong Province, Jinan 250014, China.

\section{Acknowledgements}

Not applicable.

\section{Competing interests}

The authors declare that they have no competing interests.

\section{Availability of data and materials}

Not applicable.

\section{Consent for publication}

Not applicable.

\section{Ethics approval and consent to participate}

This study was approved by the Ethics Committee of Qilu Hospital of Shandong University (KYLL-2013-097; 25 February 2014), and written informed consent was obtained from all patients or their guardians.

\section{Funding}

This work was supported by Grants from the Major Scientific and Technological Innovation Project of Shandong Province (2018CXGC1212), the Science and Technology Foundation of Shandong Province (2014GSF118084 and 2016GSF121043), the Medical and Health Technology Innovation Plan of Ji'nan City (201805002) and the National Natural Science Foundation of China (81372333).

\section{Publisher's Note}

Springer Nature remains neutral with regard to jurisdictional claims in published maps and institutional affiliations.

Received: 17 November 2018 Accepted: 14 February 2019

Published online: 18 February 2019

\section{References}

1. Bray F, Ferlay J, Soerjomataram I, Siegel RL, Torre LA, Jemal A. Global cancer statistics 2018: GLOBOCAN estimates of incidence and mortality worldwide for 36 cancers in 185 countries. CA Cancer J Clin. 2018:68:394-424.

2. Imielinski M, Berger AH, Hammerman PS, Hernandez B, Pugh TJ, Hodis E, et al. Mapping the hallmarks of lung adenocarcinoma with massively parallel sequencing. Cell. 2012;150:1107-20.

3. Siegel RL, Miller KD, Jemal A. Cancer statistics, 2018. CA Cancer J Clin. 2018;68:7-30.

4. Chen LL. The biogenesis and emerging roles of circular RNAs. Nat Rev Mol Cell Biol. 2016;17:205-11.

5. Li X, Yang L, Chen LL. The biogenesis, functions, and challenges of circular RNAs. Mol Cell. 2018;71:428-42.

6. Han B, Chao J, Yao H. Circular RNA and its mechanisms in disease: from the bench to the clinic. Pharmacol Ther. 2018;187:31-44.

7. Qiu M, Xia W, Chen R, Wang S, Xu Y, Ma Z, et al. The circular RNA circPRKCl promotes tumor growth in lung adenocarcinoma. Cancer Res. 2018;78:2839-51.

8. Wang L, Tong X, Zhou Z, Wang S, Lei Z, Zhang T, et al. Circular RNA hsa_circ_0008305 (circPTK2) inhibits TGF-beta-induced epithelial-mesenchymal transition and metastasis by controlling TIF1gamma in non-small cell lung cancer. Mol Cancer. 2018;17:140.

9. Zhang J, Liu H, Hou L, Wang G, Zhang R, Huang Y, et al. Circular RNA_LARP4 inhibits cell proliferation and invasion of gastric cancer by sponging miR-424-5p and regulating LATS1 expression. Mol Cancer. 2017; 16:151.

10. Hsiao KY, Lin YC, Gupta SK, Chang N, Yen L, Sun HS, et al. Noncoding effects of circular RNA CCDC66 promote colon cancer growth and metastasis. Cancer Res. 2017;77:2339-50.

11. Yu J, Xu QG, Wang ZG, Yang Y, Zhang L, Ma JZ, et al. Circular RNA CSMARCA5 inhibits growth and metastasis in hepatocellular carcinoma. J Hepatol. 2018;68:1214-27.

12. Han D, Li J, Wang H, Su X, Hou J, Gu Y, et al. Circular RNA circMTO1 acts as the sponge of microRNA-9 to suppress hepatocellular carcinoma progression. Hepatology. 2017;66:1151-64.

13. Chen $B$, Wei W, Huang $X$, Xie $X$, Kong $Y$, Dai D, et al. circEPSTI1 as a prognostic marker and mediator of triple-negative breast cancer progression. Theranostics. 2018;8:4003-15.

14. Rybak-Wolf A, Stottmeister C, Glazar P, Jens M, Pino N, Giusti S, et al. Circular RNAs in the mammalian brain are highly abundant, conserved, and dynamically expressed. Mol Cell. 2015;58:870-85.

15. Viereck J, Thum T. Circulating noncoding RNAs as biomarkers of cardiovascular disease and injury. Circ Res. 2017;120:381-99.

16. Li Y, Zheng Q, Bao C, Li S, Guo W, Zhao J, et al. Circular RNA is enriched and stable in exosomes: a promising biomarker for cancer diagnosis. Cell Res. 2015;25:981-4.

17. Bonizzato A, Gaffo E, Te Kronnie G, Bortoluzzi S. CircRNAs in hematopoiesis and hematological malignancies. Blood Cancer J. 2016;6:e483.

18. Tan S, Gou Q, Pu W, Guo C, Yang Y, Wu K, et al. Circular RNA F-circEA produced from EML4-ALK fusion gene as a novel liquid biopsy biomarker for non-small cell lung cancer. Cell Res. 2018;28:693-5.

19. Hang D, Zhou J, Qin N, Zhou W, Ma H, Jin G, et al. A novel plasma circular RNA circFARSA is a potential biomarker for non-small cell lung cancer. Cancer Med. 2018:7:2783-91.

20. Glazar P, Papavasileiou P, Rajewsky N. circBase: a database for circular RNAs. RNA. 2014;20:1666-70.

21. Xia S, Feng J, Chen K, Ma Y, Gong J, Cai F, et al. CSCD: a database for cancer-specific circular RNAs. Nucleic Acids Res. 2018;46:D925-9.

22. Gyorffy B, Surowiak P, Budczies J, Lanczky A. Online survival analysis software to assess the prognostic value of biomarkers using transcriptomic data in non-small-cell lung cancer. PLoS ONE. 2013;8:e82241.

23. Dudekula DB, Panda AC, Grammatikakis I, De S, Abdelmohsen K, Gorospe M. Circlnteractome: a web tool for exploring circular RNAs and their interacting proteins and microRNAs. RNA Biol. 2016;13:34-42.

24. Agarwal V, Bell GW, Nam JW, Bartel DP. Predicting effective microRNA target sites in mammalian mRNAs. Elife. 2015;4:e05005.

25. Chou CH, Shrestha S, Yang CD, Chang NW, Lin YL, Liao KW, et al. miRTarBase update 2018: a resource for experimentally validated microRNAtarget interactions. Nucleic Acids Res. 2018;46:D296-302. 
26. Doerks T, Copley RR, Schultz J, Ponting CP, Bork P. Systematic identification of novel protein domain families associated with nuclear functions. Genome Res. 2002;12:47-56.

27. da Huang W, Sherman BT, Lempicki RA. Systematic and integrative analysis of large gene lists using DAVID bioinformatics resources. Nat Protoc. 2009;4:44-57.

28. Koh W, Pan W, Gawad C, Fan HC, Kerchner GA, Wyss-Coray T, et al. Noninvasive in vivo monitoring of tissue-specific global gene expression in humans. Proc Natl Acad Sci USA. 2014;111:7361-6.

29. Bahn JH, Zhang Q, Li F, Chan TM, Lin X, Kim Y, et al. The landscape of microRNA, Piwi-interacting RNA, and circular RNA in human saliva. Clin Chem. 2015;61:221-30.

30. Beermann J, Piccoli MT, Viereck J, Thum T. Non-coding RNAs in development and disease: background, mechanisms, and therapeutic approaches. Physiol Rev. 2016;96:1297-325.

31. Li S, Teng S, Xu J, Su G, Zhang Y, Zhao J, et al. Microarray is an efficient tool for circRNA profiling. Brief Bioinform. 2018. https://doi.org/10.1093/bib/ bby006.

32. Zhu X, Wang X, Wei S, Chen Y, Chen Y, Fan X, et al. hsa_circ_0013958: a circular RNA and potential novel biomarker for lung adenocarcinoma. FEBS J. 2017;284:2170-82.

33. Li T, Shao Y, Fu L, Xie Y, Zhu L, Sun W, et al. Plasma circular RNA profiling of patients with gastric cancer and their droplet digital RT-PCR detection. J Mol Med (Berl). 2018;96:85-96.

34. Nilsson RJ, Balaj L, Hulleman E, van Rijn S, Pegtel DM, Walraven M, et al. Blood platelets contain tumor-derived RNA biomarkers. Blood. 2011;118:3680-3.

35. Lasda E, Parker R. Circular RNAs co-precipitate with extracellular vesicles: a possible mechanism for circRNA clearance. PLoS ONE. 2016;11:e0148407.

36. Shostak K, Chariot A. EGFR and NF-kappaB: partners in cancer. Trends Mol Med. 2015;21:385-93.

37. Piotrowska Z, Sequist LV. Treatment of EGFR-mutant lung cancers after progression in patients receiving first-line EGFR tyrosine kinase inhibitors: a review. JAMA Oncol. 2016;2:948-54.

38. Hirsch FR, Scagliotti GV, Mulshine JL, Kwon R, Curran WJ, Wu Y-L, et al. Lung cancer: current therapies and new targeted treatments. Lancet. 2017;389:299-311.

39. Chiou YH, Liou SH, Wong RH, Chen $\mathrm{CY}$, Lee H. Nickel may contribute to EGFR mutation and synergistically promotes tumor invasion in EGFRmutated lung cancer via nickel-induced microRNA-21 expression. Toxicol Lett. 2015;237:46-54.

40. Wu DW, Wu TC, Chen CY, Lee H. PAK1 is a novel therapeutic target in tyrosine kinase inhibitor-resistant lung adenocarcinoma activated by the PI3K/AKT signaling regardless of EGFR mutation. Clin Cancer Res. 2016;22:5370-82
41. Gong K, Guo G, Gerber DE, Gao B, Peyton M, Huang C, et al. TNF-driven adaptive response mediates resistance to EGFR inhibition in lung cancer. J Clin Invest. 2018:128:2500-18.

42. Fan Z, Yang J, Zhang D, Zhang X, Ma X, Kang L, et al. The risk variant rs884225 within EGFR impairs miR-103a-3p's anti-tumourigenic function in non-small cell lung cancer. Oncogene. 2018;23:1.

43. Hansen TB, Jensen TI, Clausen BH, Bramsen JB, Finsen B, Damgaard CK, et al. Natural RNA circles function as efficient microRNA sponges. Nature. 2013:495:384-8.

44. Memczak S, Jens M, Elefsinioti A, Torti F, Krueger J, Rybak A, et al. Circular RNAs are a large class of animal RNAs with regulatory potency. Nature. 2013:495:333-8.

45. Du W, Liu X, Chen L, Dou Z, Lei X, Chang L, et al. Targeting the SMO oncogene by miR-326 inhibits glioma biological behaviors and stemness. Neuro Oncol. 2015;17:243-53.

46. Babashah S, Sadeghizadeh M, Hajifathali A, Tavirani MR, Zomorod MS, Ghadiani M, et al. Targeting of the signal transducer Smo links microRNA-326 to the oncogenic Hedgehog pathway in CD34+CML stem/ progenitor cells. Int J Cancer. 2013;133:579-89.

47. Nawaz Z, Patil V, Paul Y, Hegde AS, Arivazhagan A, Santosh V, et al. PI3 kinase pathway regulated miRNome in glioblastoma: identification of miR-326 as a tumour suppressor miRNA. Mol Cancer. 2016;15:74.

48. Valencia K, Martin-Fernandez M, Zandueta C, Ormazabal C, MartinezCanarias S, Bandres E, et al. miR-326 associates with biochemical markers of bone turnover in lung cancer bone metastasis. Bone. 2013;52:532-9.

49. Cai M, Wang Z, Zhang J, Zhou H, Jin L, Bai R, et al. Adam17, a target of Mir326, promotes emt-induced cells invasion in lung adenocarcinoma. Cell Physiol Biochem. 2015;36:1175-85.

50. Liu H, Liu Y, Liu W, Zhang W, Xu J. EZH2-mediated loss of miR-622 determines CXCR4 activation in hepatocellular carcinoma. Nat Commun. 2015:6:8494.

51. Dietrich P, Koch A, Fritz V, Hartmann A, Bosserhoff AK, Hellerbrand C. Wild type Kirsten rat sarcoma is a novel microRNA-622-regulated therapeutic target for hepatocellular carcinoma and contributes to sorafenib resistance. Gut. 2018:67:1328-41.

52. Tong S, Xia T, Fan K, Jiang K, Zhai W, Li JS, et al. 14-3-3zeta promotes lung cancer cell invasion by increasing the Snail protein expression through atypical protein kinase C (aPKC)/NF-kappaB signaling. Exp Cell Res. 2016;348:1-9.

53. Zhao GY, Ding JY, Lu CL, Lin ZW, Guo J. The overexpression of 14-3-3zeta and Hsp27 promotes non-small cell lung cancer progression. Cancer. 2014;120:652-63.
Ready to submit your research? Choose BMC and benefit from:

- fast, convenient online submission

- thorough peer review by experienced researchers in your field

- rapid publication on acceptance

- support for research data, including large and complex data types

- gold Open Access which fosters wider collaboration and increased citations

- maximum visibility for your research: over $100 \mathrm{M}$ website views per year

At $\mathrm{BMC}$, research is always in progress.

Learn more biomedcentral.com/submissions 